\title{
The Impact of Information Environment on Enterprise Internal Control
}

\author{
Han Wang ${ }^{1, \text { a }}$ \\ ${ }^{1}$ School of Management, Xi'An Jiaotong University, Xi'An 710049, China; \\ aEmilywang58@163.com
}

Keywords: Information Environment; Enterprise Internal Control; Impact Study.

\begin{abstract}
In the information era, with the increasing risks faced by enterprises, the importance of internal control is becoming more and more obvious. However, as the internal control elements of enterprises are affected by the information environment, the traditional methods cannot meet the requirements of internal control by the enterprises. Therefore, many problems are encountered during the implementation of internal control. Based on the analysis of the difference between the internal control of information and its precedents in traditional form, this paper draws lessons from both the theory and practical experience of internal control at home and abroad, bringing forward a study toward the impact of information on the internal control elements of enterprises so as to help enterprises implement the internal control smoothly and achieve the overall goals out of internal control measures.
\end{abstract}

\section{Introduction}

In today's era of information, various aspects of the external and internal environments of enterprises are affected by the flow of information, such as organizational structures, business processes, management methods, supply chain, etc., the horizon of which have changed in accordance to the transforming landscape. The computer network interconnects the various departments, branches, suppliers and customers of enterprises. OA, ERP, SCM, CRM and other information management systems have been widely used in enterprises so that information has been integrated into all aspects of enterprises. Besides, information has also had an important impact on the various internal control elements [1]. The complex scenarios alongside with the enterprise information provide a favorable environment or condition for the implementation of internal control. The use of information management systems and other advanced means of information technology implemented within internal control measures can bring forward a more integrated control system filled with enterprise information. It can build internal control into the process of business management so as to achieve the goal of the overall control over the whole enterprise.

In the information-based environment, all the factors of internal control influenced by information have new characteristics. The original internal control theory framework cannot meet the need of enterprise's internal control. It is necessary to take full account of the information characteristics and reconstruct the internal control framework on the basis of the existing frame made by the theoretical approach. Based on the analysis of the difference between informational internal control and traditional internal control, alongside with the theories and experience of internal control at home and abroad, this paper studies the influence of information technology on the internal control elements to help enterprises implement the internal control smoothly and achieve the ultimate goal of internal control over each of enterprises’ components.

\section{Differences between Informational Internal Control and Traditional Internal Control}

\subsection{Local Control into Overall Control}

In the comprehensive information environment, enterprises treat internal control not only from the perspective of independent audit but from the perspective of business management to understand the existing problems. Gradually, Internal control has now been taken as a whole to study [2]. In addition, in the comprehensive informational environment, the information system works as a whole to achieve 
a complete business process all of which are completed online. Therefore, the internal control under the overall information environment pays more attention to the systematisms of all aspects within the control chain, and grasps the overall connotation of internal control from a higher level.

In a comprehensive information environment, the control environment has undergone significant changes, and information tools have changed the original means of controlling. Based on the agreement, the information can be shared between the various departments of enterprises, or between enterprises and customers, enterprises and regulators. This real-time monitoring and remote review are effective monitoring means and control tools in an information-based environment. In a comprehensive information environment, the internal control is no longer confined to the enterprises inside. The enterprises can use the information system to share information with customers and the supervision departments, which would gradually realize the macro-control and integration of the internal control. Therefore, in the overall information environment, internal control is not only the integration of various departments about control activities in the enterprises but also the coordination of the control activities with the correlated external institutions.

\subsection{Post-control into Real-time Control}

In the traditional enterprise environment, after various activities, there will be specialized departments organizing professional staff to carry out reviews to play a supervisory role. At this time the problems arising in business activities only can be remedied, but cannot be avoided. The traditional enterprise environment may lose the best periodic time of feedback toward the difficulties. In a comprehensive information environment, the simultaneous transmission of material flow, capital flow and information flow makes it possible that the internal control transforms from the post-control into in-process control, from timely control into real-time control.

In a comprehensive information environment, the widespread use of the network combining with the flow of internal information achieves the division of labor of the internal control and the incompatible post separation, which can not only achieve horizontal integration for the production and marketing of internal control, but also achieve the vertical internal control between the enterprise groups and their branches. Comprehensive information-based environment provides various departments within the enterprises a real-time information delivery platform. Designers, manufacturing personnels, sales staff of enterprises can enter information to the system and query information from the system simultaneously. Thus the enterprise personnels jointly control the enterprise's material flow, value flow and information flow, which translates the traditional review afterward into real-time control in advance, during and after the event.

\subsection{Command Control into Coordination Control}

At the era of traditional internal control, work can be decomposed into steps according to the specific process, and then interconnected through the command and control system. Instructions are often conveyed from the higher level to the lower level. With the introduction of internal control measures alongside with the enterprise management system, new sets of regulations and operational norms have been established. In a comprehensive information environment, businesses are able to collaborate and communicate equally and instantaneously between insiders and outsiders. Each employee is shown as a decision point or node within the enterprise control network. They are consciously involved in the control activities of the enterprise. Internal control is achieved through real-time processing and off-site review of information by employees. Therefore, in a comprehensive information environment, the enterprise's internal control transforms from its old form, which is usually carried out through command control, to the new form filled with coordination and cooperation between staffs.

\section{The Impact of Information Environment on Internal Control}

\subsection{Impact on Internal Environment}

The internal environment is the foundation of internal control, which can provide a more comprehensive and profound framework for enterprise internal control under the network environment. In the network environment, the real-time transmission of information has become a 
basic feature of enterprises. Information system has grown to be a vital part for the accounting works within companies. As a result, information and communication are no longer elements of internal control in the network environment, but important aspects of the enterprise internal environment [3]. Therefore, the internal environment of enterprise internal control in the network environment mainly includes accounting sub-rules, integrity and moral values, corporate cultures, the corporate governance mechanism, organizational structures, ERP information system, the communication mechanism and the communication system and so on. In the internal environment of network environment accounting internal control, accounting regulations are formal rules, which restricts and influences the daily behaviors of accounting organizations and accounting personnel. Honesty, moral values and corporate cultures are informal constraints, which is the code of conducts when accountants handle accounting work in a correct way, and the ideological character and the basic quality all staff engaged in the accounting profession must have. The corporate governance mechanism and organizational structure is the implementation mechanism, which is a powerful tool to ensure the implementation of accounting internal control. ERP namely the information system is the operating environment and platform of the accounting internal control and the basis and guarantee of the effective execution of accounting internal control. In the network environment, the implementation of ERP makes the enterprise's organizational structure show a flat and process-oriented character, the internal control levels of the enterprise obviously reduced. Roles and operator authorizations make accountability more explicit and control efficiency higher. In the meanwhile, it enhances the flexibility of enterprise internal control because the system can achieve the cross-authorization of the operators easily.

\subsection{Impact on Risk Assessment}

In modern enterprises, the risk is everywhere. Risk management has become the key to improve the efficiency and effectiveness of accounting internal control. Controlling measures toward risks have also become the important content of frame constitution about the enterprise accountant internal control. Under the network environment, in the framework of accounting internal control, risk management includes three sub-elements: risk identification, risk assessment and risk coping, which constitutes the third level of the accounting internal control framework. In the network environment, in addition to identifying and assessing internal and external business process risk, financial risk and regulatory risk, it is more important for accounting internal control to identify and evaluate the new risks caused by information systems, including the management risk of information system planning and construction, internal control mechanism vulnerability risk about software, unstable risks on operation of the system, the risk of man-made operations and so on. Whether we pay attention to strengthening the identification and evaluation of the risks within the information system and its result is the key to decide whether we can achieve the success of accounting internal control in the network environment.

Enterprise information norms solidify the business process and system control, meanwhile, reduces the risks of omissions or frauds. The potential risks toward information storage are often reflected regarding the highly-centralized characteristic of the internal control system, which may lead to system failures, crashes and data theft. We need to establish some good IT governance mechanisms to prevent disasters and reduce the loss.

\subsection{Impact on Control activities}

Control activities are that the risks can be controlled within tolerance based on the results of risk assessment and by means of appropriate control measures. Control activities should be based on the results of risk assessment and set specific control points in the business process through implementing appropriate control measures.

In the information environment, the enterprise's organizational structure adjusted and the business process restructured must have an impact on internal control activities. With the help of advanced information tools and information systems, control activities gradually change from the traditional manual way to automatic control. Through the automatic control of information system, the degree of human involvement can be reduced, which greatly reduces the occurrence of human errors and artificial frauds and can improve the efficiency of control activities. In addition, the use of advanced 
information systems can be preventive and a real-time control for some business so as to help enterprises deal with the risks and avoid losses in time. With the wide application of various information systems in the enterprise, the objects of control activities in addition to production, management and operation, should also include various types of information systems. Controlling information systems not only ensures the operation of information systems normal and safe, but also prevents all types of information technology risks.

\subsection{Impact on Internal supervision}

Internal supervision is the supervision and inspection toward the establishment and implementation of internal control, which is to evaluate the effectiveness of internal control. If deficiencies of internal control are found, they should be improved in time. Enterprise internal control is a dynamic closed-loop system, including the design, implementation, evaluation and improvement four links of internal control. This requires internal supervision to assess the effectiveness of internal control. Compared with the goal of internal control, the feedback can be gotten from the gap between them in order to improve internal control.

In the information-based environment, by means of specialized information systems, you can achieve the internal supervision process automation in a degree. What's more, it can achieve real-time monitoring to enhance the effectiveness and efficiency of internal supervision. Control Self-Assessment (CSA) is an important method to test and evaluate the effect of internal control, which should be integrated into the internal control information system. With the help of the information system, real-time supervision can be realized so as to improve the efficiency and efficiency of supervision. The supervision of development, implementation and maintenance to information system should be the focus. The enterprise evaluates the internal control system on a regular or irregular basis so as to evaluate the effectiveness of the system and its implementation, which is to achieve the goals of internal control better.

\section{Conclusions}

In this paper, first of all, we analyze the differences of internal control between under the information environment and the traditional environment. Then we discuss the influence of information environment on enterprise internal control. Through the analysis above, we can conclude that, enterprise information can create a good internal environment for internal control and provide advanced technical means for the implementation of internal control, which is conducive to improving the efficiency and effectiveness of internal control in order that the goal of internal control can be achieved at last.

\section{References}

[1] Lee, T. A. (1971). The historical development of internal control from the earliest times to the end of the seventeenth century. Journal of Accounting Research, 150-157.

[2] Root, S. J., \& Grumman, N. (1998). Beyond COSO: internal control to enhance corporate governance. New York, NY: Wiley.

[3] Hoitash, U., Hoitash, R., \& Bedard, J. C. (2009). Corporate governance and internal control over financial reporting: A comparison of regulatory regimes.The accounting review, 84(3), 839-867.

[4] Cheng, M., Dhaliwal, D., \& Zhang, Y. (2013). Does investment efficiency improve after the disclosure of material weaknesses in internal control over financial reporting. Journal of Accounting and Economics, 56(1), 1-18. 